\title{
New instability for rotating black branes and strings
}

\author{
Vitor Cardoso $^{\mathrm{a}, \mathrm{b}}$, José P.S. Lemos ${ }^{\mathrm{c}}$ \\ a McDonnell Center for the Space Sciences, Department of Physics, Washington University, St. Louis, MO 63130, USA \\ ${ }^{\mathrm{b}}$ Centro de Física Computacional, Universidade de Coimbra, P-3004-516 Coimbra, Portugal \\ ${ }^{\mathrm{c}}$ Centro Multidisciplinar de Astrofísica (CENTRA), Departamento de Física, Instituto Superior Técnico, Universidade Técnica de Lisboa, \\ Av. Rovisco Pais 1, 1049-001 Lisboa, Portugal
}

Received 20 May 2005; accepted 9 June 2005

Available online 20 June 2005

Editor: M. Cvetič

\begin{abstract}
The evolution of small perturbations around rotating black branes and strings, which are low energy solutions of string theory, are investigated. For simplicity, we concentrate on the Kerr solution times transverse flat extra dimensions, possibly compactified, but one can also treat other branes composed of any rotating black hole and extra transverse dimensions, as well as analogue black hole models and rotating bodies in fluid mechanics systems. It is shown that such a rotating black brane is unstable against any massless (scalar, vectorial, tensorial or other) field perturbation for a wide range of wavelengths and frequencies in the transverse dimensions. Since it holds for any massless field it can be considered, in this sense, a stronger instability than the one studied by Gregory and Laflamme. Accordingly, it has also a totally different physical origin. The perturbations can be stabilized if the extra dimensions are compactified to a length smaller than the minimum wavelength for which the instability settles in, resembling in this connection the Gregory-Laflamme case. Likewise, this instability will have no effect for astrophysical black holes. However, in the large extra dimensions scenario, where TeV scale black holes can be produced, this instability should be important. It seems plausible that the endpoint of this instability is a static, or very slowly rotating, black brane and some outgoing radiation at infinity.
\end{abstract}

(c) 2005 Elsevier B.V. All rights reserved.

PACS: 04.50.+h; 04.70.Bw; 11.25.-w; 11.27.+d

\section{Introduction}

A consistent theory of quantum gravity, such as string theory, seems to require the existence of higher

E-mail addresses: vcardoso@wugrav.wustl.edu (V. Cardoso), lemos@fisica.ist.utl.pt (J.P.S. Lemos). dimensions, which in order not to contradict observational evidence must be compactified on small scales. String theory has made some important progress in explaining the entropy of certain black holes by counting microscopic degrees of freedom, and thus scenarios with extra, compactified dimensions must be taken seriously. In turn, some of the most interesting objects to be studied within string theory are those that possess 
event horizons, possibly extended in the compact extra dimensions.

In $d$ spacetime dimensions, an event horizon can be topologically a sphere $S^{d-2}$, but when extended to $p$ extra dimensions, it could naturally have topologies either $S^{d-2+p}$ in which case it is a higher-dimensional black hole, or $S^{d-2} \times R^{p}$ being then a black $p$-brane or a black string in the $p=1$ case [1]. The $R^{p}$ topology of the transverse dimensions can be compactified giving flat toroidal topological spaces $T^{p}$.

One of the first steps towards understanding these extended higher-dimensional solutions is to investigate their classical stability against small perturbations. If a solution is unstable, then it most certainly will not be found in nature (unless the instability is secular) and the solution looses most of its power. Of course the next question one must ask is what is the final stable result of such instability. Now, in $d$ spacetime dimensions, the Schwarzschild-Tangherlini geometry is stable against all kinds of perturbations, massive or massless [2]. On the other hand, quite surprisingly, Gregory and Laflamme [3] showed that this is not the case for higher-dimensional black branes and black strings. These objects are unstable. The kind of black $p$-branes originally studied in [3] were solutions of ten-dimensional low energy string theory with metric of the form

$d s^{2}=d s_{\text {Schw }}^{2}+d x^{i} d x_{i}$,

where $d s_{\text {Schw }}^{2}$ stands for the $d$-dimensional Schwarzschild-Tangherlini line element, the $x^{i}$ are the coordinates of the compact dimensions, and $i$ runs from 1 to $p$. The total dimension of the spacetime $D$ obeys $D=d+p$. In string or supergravity theories one takes $D=10$ or $D=11$, but for genericity one can leave it as a free natural parameter. In [3] it was shown that even though scalar and vector perturbations of the black brane stay bounded in time, the tensorial sector of gravitational perturbations displays an instability, with the perturbations growing exponentially with time, possibly breaking the extended black brane into several smaller black holes. The only way around this instability is to compactify the transverse directions $x^{i}$ on a scale smaller than the black hole radius [3] (for a full discussion of this instability and its developments see [4]).
Here we shall show that a related class of metrics, for example, having the form

$d s^{2}=d s_{\mathrm{Kerr}}^{2}+d x^{i} d x_{i}$,

where the line element $d s_{\text {Kerr }}^{2}$ stands for the $d$-dimensional Kerr-Myers-Perry line element, is unstable to scalar perturbations, as well as to vectorial, gravitational and other type of perturbations. In this sense it is a stronger instability than the one studied in [3], because it holds for any massless field. Accordingly, it has also a totally different physical origin, as we will see.

\section{Formulation of the problem and basic equations}

\subsection{The background metric}

In four dimensions, there is only one possible rotation axis for a cylindrically symmetric spacetime, and there is therefore only one angular momentum parameter. In higher dimensions there are several choices for rotation axes and there is a multitude of angular momentum parameters, each referring to a particular rotation axis. Here we shall concentrate on the simplest case, for which there is only one angular momentum parameter, which we shall denote by $a$. The specific metric we shall be interested in is given in Boyer-Lindquist-type coordinates by

$$
\begin{aligned}
d s^{2}= & -\frac{\Delta-a^{2} \sin ^{2} \theta}{\Sigma} d t^{2} \\
& -\frac{2 a\left(r^{2}+a^{2}-\Delta\right) \sin ^{2} \theta}{\Sigma} d t d \varphi \\
& +\frac{\left(r^{2}+a^{2}\right)^{2}-\Delta a^{2} \sin ^{2} \theta}{\Sigma} \sin ^{2} \theta d \varphi^{2} \\
& +\frac{\Sigma}{\Delta} d r^{2}+\Sigma d \theta^{2}+r^{2} \cos ^{2} \theta d \Omega_{n}^{2}+d x^{i} d x_{i},
\end{aligned}
$$

where

$$
\begin{aligned}
& \Sigma=r^{2}+a^{2} \cos ^{2} \theta, \\
& \Delta=r^{2}+a^{2}-m r^{1-n},
\end{aligned}
$$

and $d \Omega_{n}^{2}$ denotes the standard metric of the unit $n$ sphere $(n=d-4)$, the $x^{i}$ are the coordinates of the compact dimensions, and $i$ runs from 1 to $p$. This 
metric describes a rotating black brane in an asymptotically flat, vacuum space-time with mass and angular momentum proportional to $\mu$ and $\mu a$, respectively. Hereafter, $\mu, a>0$ are assumed.

The event horizon, homeomorphic to $S^{2+n}$, is located at $r=r_{+}$, such that $\left.\Delta\right|_{r=r_{+}}=0$. For $n=0$, an event horizon exists only for $a<\mu / 2$. When $n=1$, an event horizon exists only when $a<\sqrt{\mu}$, and the event horizon shrinks to zero-area in the extreme limit $a \rightarrow \sqrt{\mu}$. On the other hand, when $n \geqslant 2, \Delta=0$ has exactly one positive root for arbitrary $a>0$. This means there is no bound on $a$, and thus there are no extreme Kerr black branes in higher dimensions.

\subsection{Separation of variables and boundary conditions}

Consider now the evolution of a massless scalar field $\Psi$ in the background described by (3). The evolution is governed by the curved space Klein-Gordon equation

$$
\frac{\partial}{\partial x^{\mu}}\left(\sqrt{-g} g^{\mu \nu} \frac{\partial}{\partial x^{\nu}} \Psi\right)=0,
$$

where $g$ is the determinant of the metric. The metric appearing in (6) should describe the geometry referring to both the black brane and the scalar field, but if we consider that the amplitude of $\Psi$ is so small that its contribution to the energy content can be neglected, then the metric (3) should be a good approximation to $g_{\mu \nu}$ in (6). We shall thus work in this perturbative approach. It turns out that it is possible to simplify considerably equation (6) if we separate the angular variables from the radial and time variables, as is done in four dimensions [5]. For higher dimensions we follow [6]. In this connection see also [7] for a general $(4+n)$-dimensional Kerr hole with several spin parameters. In the end our results agree with the results in [7], if we consider only one angular momentum parameter in their equations, and no extra compactified dimensions.

We consider the ansatz

$\phi=\mathrm{e}^{i \omega t-i m \varphi+i k_{i} x^{i}} R(r) S(\theta) Y(\Omega)$,

and substitute this form in (6), where $Y(\Omega)$ are hyperspherical harmonics on the $n$-sphere, with eigenvalues given by $-j(j+n-1)(j=0,1,2, \ldots)$. Then we ob- tain the separated equations

$$
\begin{aligned}
& \frac{1}{\sin \theta \cos ^{n} \theta}\left(\frac{d}{d \theta} \sin \theta \cos ^{n} \theta \frac{d S}{d \theta}\right) \\
& +\left[a^{2}\left(\omega^{2}-k^{2}\right) \cos ^{2} \theta-m^{2} \csc ^{2} \theta\right. \\
& \left.-j(j+n-1) \sec ^{2} \theta+A\right] S=0,
\end{aligned}
$$

and

$$
\begin{aligned}
r^{-n} & \frac{d}{d r}\left(r^{n} \Delta \frac{d R}{d r}\right) \\
& +\left\{\frac{\left[\omega\left(r^{2}+a^{2}\right)-m a\right]^{2}}{\Delta}\right. \\
& \left.-\frac{j(j+n-1) a^{2}}{r^{2}}-\lambda-k^{2} r^{2}\right\} R=0,
\end{aligned}
$$

where $A$ is a constant of separation, $\lambda:=A-2 m \omega a+$ $\omega^{2} a^{2}$, and $k^{2}=\sum k_{i}^{2}$. Interestingly, note the important point that Eqs. (7) and (8) are just those that describe the evolution of a massive scalar field, with mass $k$, in a $d$-dimensional Kerr geometry.

Eqs. (7) and (8) must be supplemented by appropriate boundary conditions, which are given by

$$
R \sim \begin{cases}\left(r-r_{H}\right)^{i \sigma} & \text { as } r \rightarrow r_{H}, \\ r^{-(n+2) / 2} \mathrm{e}^{-i \sqrt{\omega^{2}-k^{2}} r} & \text { as } r \rightarrow \infty,\end{cases}
$$

where

$$
\sigma:=\frac{\left[\left(r_{H}^{2}+a^{2}\right) \omega-m a\right] r_{H}}{(n-1)\left(r_{H}^{2}+a^{2}\right)+2 r_{H}^{2}}
$$

has been determined by the asymptotic behavior of Eq. (8). In other words, the waves must be purely ingoing at the horizon and purely outgoing at the infinity. For assigned values of the rotational parameter $a$ and of the angular indices $l, j, m$ there is a discrete (and infinite) set of frequencies called quasinormal frequencies, $\mathrm{QN}$ frequencies or $\omega_{\mathrm{QN}}$, satisfying the wave equation (8) with the boundary conditions just specified by Eq. (9).

\section{The instability timescale}

Now, when $d=4$, analysis of the perturbations of a massive scalar field on the Kerr geometry has been done [8]. Indeed, Eqs. (7) and (8) have been analytically solved by Zouros and Eardley in the limit of large $k^{2}$ and by Detweiler in the limit of small $k^{2}$ [8]. 
Detweiler's results have recently been confirmed numerically by Furuhashi and Nambu [8]. Detweiler [8] shows that for small $k^{2}$, in particular, $k M \ll 1$, the geometry is unstable. In fact, he shows that the characteristic frequencies $\omega$ satisfying the boundary conditions (9) are given by

$\omega=\sigma+i \gamma$,

with

$\sigma^{2}=k^{2}\left[1-\left(\frac{k M}{l+1+n}\right)^{2}\right], \quad n=0,1,2, \ldots$,

and

$\gamma=C\left(k-\frac{a m}{2 M r_{+}}\right)$

with $C=C(l, m, a, M, n)$ a positive constant, as shown in Detweiler [8]. So, for $m$ positive and

$k<\frac{a m}{2 M r_{+}}$

which is just a superradiance condition, the mode is then unstable. In particular, the most unstable mode is the $l=m=1$ mode with an $e$-folding time $\tau$ given by of

$$
\frac{\tau}{M}=24 \frac{1}{k a} \frac{1}{(k M)^{8}} \text {. }
$$

Now, we know their results can be translated immediately into our black brane geometry. Thus rotating black branes of the form (3) with $d=4$ and $p$ extra dimensions are unstable, with an $e$-folding time given by (15). The results for $d>4$ are not available in the literature, but the instability should be present in these cases as well.

The only way around this instability is to make the superradiant factor $k-\frac{a m}{2 M r_{+}}$positive, which is the same as having $k M>\frac{a m}{2 r_{+}}$. Thus, for sufficiently small $a / r_{+}$, this is still inside the range of validity of our approximations, $k M \ll 1$. To escape this instability one must compactify the transverse dimensions on a small scale $L$, i.e., $\frac{L}{2 \pi M}<\frac{2 r_{+}}{a}$. Writing out $r_{+}$explicitly this gives

$\frac{L}{2 \pi M}<2 \alpha$,

where $\alpha=M / a+\sqrt{M^{2} / a^{2}-1} \sim 2 M / a$, for small $a$. From (15) one obtains that the timescale for the instability, in the approximations used is $\tau / M>$ $24(1 / k M)^{9}$. Since $k M \ll 1$ this is not a specially efficient instability, and it does not even appear to overcome the Gregory-Laflamme instability timescale. However, the only results available so far for the Gregory-Laflamme instability refer to non-rotating objects. It is possible that as one increases the rotation to large values the instability studied here becomes more effective (as is apparent from Eq. (15)) and that the Gregory-Laflamme instability gets less effective.

\section{Conclusions}

What is the physical interpretation of this instability for rotating black branes? It is known that the Kerr geometry displays superradiance [9]. This means that in a scattering experiment of a wave with frequency $\omega<m \Omega$ the scattered wave will have a larger amplitude than the incident wave, the excess energy being withdrawn from the object's rotational energy. Here $\Omega$ is the horizon's angular velocity (related to $a, M$ and $r_{+}$through $\Omega=a /\left(2 M r_{+}\right)$) and $m$ is again the azimuthal wave quantum number. Now suppose that one encloses the rotating black hole inside a spherical mirror. Any initial perturbation will get successively amplified near the black hole event horizon and reflected back at the mirror, thus creating an instability. This is the black hole bomb, as devised in [10] and recently improved in [11]. This instability is caused by the mirror, which is an artificial wall, but one can devise natural mirrors if one considers massive fields. In this case, the mass of the field acts effectively as a mirror, and thus Kerr black holes are unstable against massive field perturbations [8]. With this in mind, we expect that black strings and branes of the form (2) will be unstable, because the compactified transverse directions work as an effective mass for the graviton and for the scalar field. In fact, this simple reasoning implies that any rotating black brane (more general than the one described by (2)) will be unstable. This would be the case for the rotating black hole in string theory found by Sen or other rotating black holes [12]. Moreover, following Zel'dovich [9], it is known that not only the Kerr geometry, but any rotating absorbing body for that matter displays superradiance. Thus, this instability should appear in analogue black hole models [13], and in rotating bodies in fluid mechanics systems. 
Here, we have derived the instability timescale for a scalar field, and not for geometry (metric) perturbations, since Teukolsky's formalism for higherdimensional rotating objects is not available. Still, the argument presented above makes it clear that the instability should be present for metric perturbations as well. We also expect that the instability will be stronger for metric modes, because of the following simple reasoning. Superradiance is the mechanism responsible for this instability, and thus the larger the superradiant effects, the stronger the instability. Now, we know that in the Kerr geometry in $d=4$ scalar fields have a maximum superradiant amplification factor of about $2 \%$, whereas gravitational modes have maximum superradiant amplification factor of about $138 \%$ [14]. Then it is expected the instability timescale to be almost two orders of magnitude smaller, and correspondingly, the instability should grow much stronger for gravitational modes. For general black branes, the Gregory-Laflamme instability seems to be stronger than the one displayed here, but it is known that certain extremal solutions should not exhibit the Gregory-Laflamme instability [15], whereas the instability dealt with here should go all the way to extremality. So, eventually it takes over the Gregory-Laflamme instability. Moreover, recent studies [16] seem to indicate that black strings in a Randall-Sundrum inspired 2-brane model do not exhibit the Gregory-Laflamme instability.

The endpoint of this rotating instability is not known, and it can never be predicted with certainty by a linear analysis. However, it seems plausible to assume that the instability will keep growing until the energy and angular momentum content of the field approaches that of the black brane, when back-reaction effects become important. The rotating brane will then begin to spin down, and gravitational and scalar radiation goes off to infinity carrying energy and angular momentum. The system will probably asymptote to a static, or very slowly rotating, final state consisting of a non-rotating black $p$-brane and some outgoing radiation at infinity. But we cannot discard the other possibility that the horizon fragments.

\section{Acknowledgements}

We gratefully acknowledge stimulating correspondence on this problem with Donald Marolf. V.C. ac- knowledges financial support from Fundaçao para a Ciência e Tecnologia (FCT), Portugal, through grant SFRH/BPD/2003. This work was partially funded by FCT through project POCTI/FNU/44648/2002.

\section{References}

[1] F.R. Tangherlini, Nuovo Cimento 27 (1963) 636; R.C. Myers, M.J. Perry, Ann. Phys. 172 (1986) 304; G.T. Horowitz, A. Strominger, Nucl. Phys. B 360 (1991) 197.

[2] T. Regge, J.A. Wheeler, Phys. Rev. 108 (1957) 1063; H. Kodama, A. Ishibashi, Prog. Theor. Phys. 110 (2003) 701; H. Kodama, A. Ishibashi, Prog. Theor. Phys. 110 (2003) 901; H. Kodama, A. Ishibashi, Prog. Theor. Phys. 111 (2004) 29; V. Cardoso, O.J.C. Dias, J.P.S. Lemos, Phys. Rev. D 67 (2003) 064026;

R.A. Konoplya, Phys. Rev. D 68 (2003) 124017; R.A. Konoplya, Phys. Rev. D 68 (2003) 024018; E. Berti, M. Cavaglia, L. Gualtieri, Phys. Rev. D 69 (2004) 124011.

[3] R. Gregory, R. Laflamme, Phys. Rev. Lett. 70 (1993) 2837.

[4] B. Kol, hep-th/0411240.

[5] D.R. Brill, P.L. Chrzanowski, C.M. Pereira, E.D. Fackerell, J.R. Ipser, Phys. Rev. D 5 (1972) 1913; S.A. Teukolsky, Phys. Rev. Lett. 29 (1972) 1114.

[6] D. Ida, Y. Uchida, Y. Morisawa, Phys. Rev. D 67 (2003) 084019

[7] V.P. Frolov, D. Stojkovic, Phys. Rev. D 67 (2003) 084004; M. Vasudevan, K.A. Stevens, D.N. Page, gr-qc/0407030.

[8] T. Damour, N. Deruelle, R. Ruffini, Lett. Nuovo Cimento 15 (1976) 257;

T.M. Zouros, D.M. Eardley, Ann. Phys. 118 (1979) 139;

S. Detweiler, Phys. Rev. D 22 (1980) 2323;

H. Furuhashi, Y. Nambu, gr-qc/0402037;

M.J. Strafuss, G. Khanna, gr-qc/0412023.

[9] Ya.B. Zel'dovich, Pis'ma Zh. Eksp. Teor. Fiz. 14 (1971) 270, JETP Lett. 14 (1971) 180;

Ya.B. Zel'dovich, Zh. Eksp. Teor. Fiz. 62 (1972) 2076, Sov. Phys. JETP 35 (1972) 1085;

C.W. Misner, Phys. Rev. Lett. 28 (1972) 994;

J. Bekenstein, Phys. Rev. D 7 (1973) 949;

W. Unruh, Phys. Rev. D 10 (1974) 3194.

[10] W.H. Press, S.A. Teukolsky, Nature 238 (1972) 211.

[11] V. Cardoso, O.J.C. Dias, J.P.S. Lemos, S. Yoshida, Phys. Rev. D 70 (2004) 044039;

V. Cardoso, O.J.C. Dias, Phys. Rev. D 70 (2004) 084011.

[12] A. Sen, Phys. Rev. Lett. 69 (1992) 1006; M. Cvetič, D. Youm, Nucl. Phys. B 476 (1996) 118.

[13] V. Cardoso, in preparation.

[14] S.A. Teukolsky, W.H. Press, Astrophys. J. 193 (1974) 443.

[15] S.H. Reall, Phys. Rev. D 64 (2001) 044005.

[16] S.S. Seahra, C. Clarkson, R. Maartens, gr-qc/0408032; See also: E. Berti, K.D. Kokkotas, E. Papantonopoulos, Phys. Rev. D 68 (2003) 064020. 